Check for updates

Cite this: RSC Adv., 2018, 8, 21863

\title{
Evolution of molecular composition of polycarbosilane and its effect on spinnability $\uparrow$
}

\author{
Wang Guodong, (D) Song Yongcai* and Li Yongqiang
}

The molecular composition of polycarbosilane (PCS) and its evolution in the synthesis process, as well as the contribution of each component to the spinnability of PCS, were explored by combining distillation separation with gel permeation chromatogram peak-splitting. The results show that PCS is composed of four kinds of molecules with different structures, namely $M_{L}, M_{M}, M_{H}$, and $M_{S H}$ (number-average molecular weights of 531, 1207, 2731, and 10 977, respectively). In the synthesis process, PCS changed from containing two components to three components, and finally to four components. The PCS can be spun when it is composed of $M_{L}, M_{M}$, and $M_{H}$, but is not suitable for spinning when it is composed of one, two, or four components. For a PCS having three components, increasing the amount of $M_{H}$ and restricting $M_{L}$ to within a certain range can enhance the spinnability of PCS. The underlying mechanism is controlled by the linear structure of $M_{H}$ and its low melting point by there being an appropriate $M_{L}$ content, which ensures melting, de-foaming, and stability in spinning. The final optimum ratio is found to be an $M_{H}$ content of $30-45 \%$, an $M_{M}$ content of $30-51 \%$, and an $M_{L}$ content of $18-25 \%$.

Received 8th March 2018

Accepted 4th June 2018

DOI: $10.1039 / \mathrm{c} 8 \mathrm{ra02045 \textrm {k }}$

rsc.li/rsc-advances

researchers' attention. Based on the solvent-extraction method

\section{Introduction}

Silicon carbide (SiC) fibres are widely employed in industry as reinforcements for advanced ceramic-matrix composites (CMCs), due to their high resistance to oxidation and corrosion, high specific strength/modulus, low density, etc. ${ }^{1-6}$ They are generally derived from polycarbosilane (PCS), the precursor to SiC, via melt spinning, curing, and high-temperature pyrolysis. Therefore, given their importance as a raw material, the relationship between the molecular structure, molecular weight distribution, and spinnability of PCS is an important issue affecting the preparation of high-performance SiC fibres.

PCS is a polymer with a certain molecular weight distribution. In early studies, ${ }^{6,7}$ PCS was studied as a whole. It was thought that the number average molecular weight $\left(M_{\mathrm{n}}\right)$ and softening point of PCS increased with the preparation temperature or time, while the molecular weight distribution changed from a single peak to a double peak. When the preparation temperature or time was further increased, a peak corresponding to super-high-molecular-weight molecules appeared. This component proved detrimental to the spinnability. The evolution of the molecular weight distribution indicated that PCS is made up of many molecules, each having a different $M_{\mathrm{n}}$. Therefore, the relationship between the molecular composition and spinning performance of PCS has become the focus of

Science and Technology on Advanced Ceramic Fibers and Composites Laboratory, National University of Defense Technology, Changsha 410073, P. R. China. E-mail: songyongcai_gy@163.com; Tel: +8613808436808

$\dagger$ Electronic supplementary information (ESI) available. See DOI: $10.1039 / \mathrm{c} 8 \mathrm{ra02045 \textrm {k }}$ or chromatographic-column grading method, ${ }^{8}$ PCS was divided into multiple components according to the experimental conditions. As the $M_{\mathrm{n}}$ increased, so too did the degree of branching, while the Si-H content decreased. Those components with an initial melting point of less than $200{ }^{\circ} \mathrm{C}$ can be spun, while the other components proved difficult or even impossible to spin. In the above studies, the PCS was separated by adjusting the solvent ratio or the elution time. The results were influenced by the experimental conditions and did not reflect the actual molecular composition of the PCS. Therefore, the relationship between the spinnability and the molecular composition of the PCS was not accurately investigated. In other studies, ${ }^{9,10}$ based on the evolution of the molecular weight distribution of the PCS, the PCS was divided into high-, medium-, and low-molecular-weight components by drawing lines across the gel permeation chromatograph (GPC). With the condensation reaction carried out, the branching and ring structure of molecules increase, and the ratio of high molecular weight components is increasing too. Only PCS containing less high-molecular-weight component and more low-molecularweight component has good spinnability. In the above research, the relationship between molecular weight distribution and spinnability of PCS was initially revealed, but the PCS was divided into different components empirically, then different researchers give different standards. Therefore, the conclusion are quite different and may be inaccurate.

The spinnability of the PCS is closely related to its structure and molecular composition. Therefore, it is very important to accurately determine the molecular composition of the PCS, as 
well as study the relationship between the composition, structure and spinnability of the PCS when preparing highperformance SiC fibres. To overcome the above problems, in the present study we used, for the first time, distillation separation combined with GPC chromatographic analysis, to test and analyse a number of samples. The molecular composition of the PCS and the evolution of the synthesis process were studied systematically. The influence of the mechanism on the spinning performance was discussed.

\section{Experimental}

\subsection{Raw materials and reagents}

Polydimethylsilane (PDMS) (purity $>98 \%$ ) was obtained from Shenzhen Gujia Chemicals Co., Ltd., while xylene (purity > 99\%) was sourced from Tianjin Hengxing Chemical Reagent Co., Ltd.

\subsection{PCS synthesis and separation}

PDMS was placed in a three-neck flask fitted with a condenser and receiving trap, for pyrolysis at $400{ }^{\circ} \mathrm{C}$ in a protective $\mathrm{N}_{2}$ atmosphere. Liquid polysilane (LPS) was obtained after the resulting solution was cooled and filtered..$^{6,9,10}$

The obtained LPS was placed in a three-neck flask fitted with a quartz reactor tube, a condenser, and a receiving trap. The LPS was then heated to $450{ }^{\circ} \mathrm{C}$ in a protective $\mathrm{N}_{2}$ atmosphere. This temperature was maintained for a few hours, after which the solution was cooled to room temperature to obtain pre-PCS. The pre-PCS was dissolved in xylene, filtered, and vacuum-distilled at $350{ }^{\circ} \mathrm{C}$ for $10 \mathrm{~min}$. Finally, the reactor was cooled to room temperature to obtain PCS.

The PCS was placed in a tilted test tube, heated to a temperature of $<450{ }^{\circ} \mathrm{C}$ in a sand bath under $\mathrm{N}_{2}$ protection, distilled under reduced pressure for some time, and then cooled to room temperature to obtain the different components of PCS.

\subsection{Spinnability of PCS}

Approximately $10 \mathrm{~g}$ of PCS was placed in a single-hole meltspinning apparatus, heated to $140-160{ }^{\circ} \mathrm{C}$ above the initial melting point for $2 \mathrm{~h}$ to allow it to de-foam, and then cooled to the spinning temperature. The spinning pressure was set to $0.2-$ $0.3 \mathrm{MPa}$ and the spinning speed to $240-294 \mathrm{~m} \mathrm{~min}^{-1}$. With continuous spinning time $(t)$ as the criterion of spinnability: $t>$ $30 \mathrm{~min}$, spinnability is excellent; $15 \mathrm{~min}<t<30 \mathrm{~min}$, spinnability is good; $5 \mathrm{~min}<t<15 \mathrm{~min}$, spinnability is moderate; $t<$ $5 \mathrm{~min}$, spinnability is poor. Meanwhile, as the spun PCS fibre becomes finer, the spinnability of the raw PCS is said to be better. $^{10}$

\subsection{Analysis and characterisation}

The softening points of the samples were measured using a Shanghai Precision Scientific Instrument WRS-2A microprocessor melting-point apparatus, with a heating rate of $2{ }^{\circ} \mathrm{C} \mathrm{min}^{-1}$. The $M_{\mathrm{n}}$ and distribution of the molecular weights of the samples were measured using a Wyatt Technology DAWN HELEOS-II high-performance gel permeation chromatograph
(GPC), with an s-Styragel column, and tetrahydrofuran (THF) as

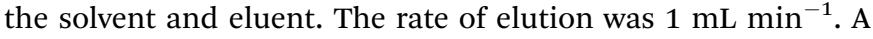
three-dimensional (3D) super-depth digital microscope (VHX500 , Keyence, Japan) was used to capture photos of fibers and to calculate the average diameter of 50 fibers.

\section{Results and discussion}

\subsection{Composition of PCS}

The PCS used for the commercial preparation of SiC fibre was fabricated using a PDMS pyrolysis rearrangement transformation method. ${ }^{11}$ This method is simple and can be easily applied to mass-production. The $M_{\mathrm{n}}$ of the PCS and its structure are closely related to its synthesis conditions. PCS (hereinafter referred to as PCS-10) with the same molecular weight distribution and softening point as PCS used for the commercial preparation of SiC fibre was synthesised in the laboratory according to the above process and reacted at $450{ }^{\circ} \mathrm{C}$ for $10 \mathrm{~h}$. The softening point was found to be $216-241{ }^{\circ} \mathrm{C}$. The $M_{\mathrm{n}}$ was 1758 . The GPC curve exhibited a "bimodal" distribution, as shown in Fig. 1.

Due to the different synthesis conditions of the samples, when using the line-drawing method described in the literature, only one boundary can be drawn, at an elution time of about $13.2 \mathrm{~min}$, thus dividing the PCS-10 into two parts. ${ }^{11}$ However, there is an obvious bulge in the figure at about $t=15.5 \mathrm{~min}$, indicating that there should actually be three regions of concentration for the PCS-10. Since the PCS synthesis reaction is a free radical reaction, the intermolecular reaction combination in the reaction process is random. Therefore, the distribution of each component should be a normal distribution. According to the above speculation, the XPSPEAK software was used to divide the GPC curve for the PCS-10 into three normal distributions of the molecular peaks shown in Fig. 1. The fitted curve for the three peaks exactly matches the original GPC curve, indicating that the PCS-10 is indeed composed of three kinds of molecule, with different $M_{\mathrm{n}}$ and structures. The peak values corresponding to each component were 15.3, 14.0, and $12.4 \mathrm{~min}$, while the $M_{\mathrm{n}}$ were 531, 1207, and 2731, respectively. According to the $M_{\mathrm{n}}$, from low to high, the molecules corresponding to the three peaks were recorded as $\mathrm{M}_{\mathrm{L}}, \mathrm{M}_{\mathrm{M}}$, and $\mathrm{M}_{\mathrm{H}}$, respectively. The mass ratio of each component was obtained by integrating the area as $\mathrm{M}_{\mathrm{L}}: \mathrm{M}_{\mathrm{M}}: \mathrm{M}_{\mathrm{H}}=$ $0.19: 0.37: 0.45$.

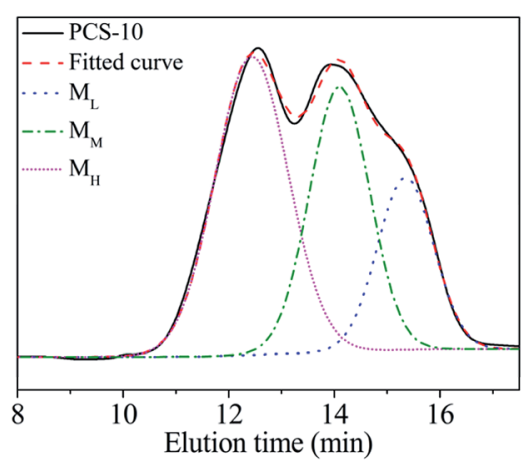

Fig. 1 GPC curve and fitting curves for PCS-10. 


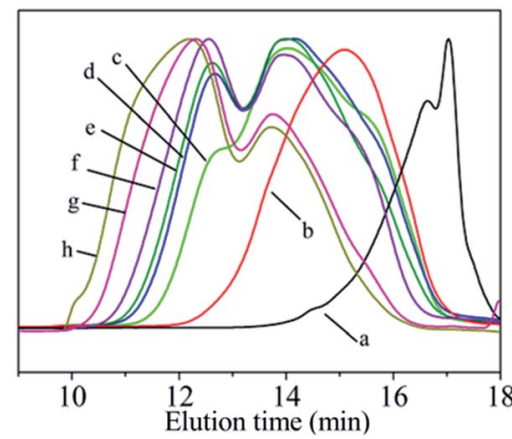

Fig. 2 GPC curves of PCS synthesised under different conditions. (a) LPS; (b) LPCS; (c) PCS-2; (d) PCS-6; (e) PCS-8; (f) PCS-10; (g) PCS-16; (h) PCS-24.

Table 1 Properties of PCS synthesised under different conditions

\begin{tabular}{llllll}
\hline & $\begin{array}{l}\text { Reaction } \\
\text { temperature } \\
\left({ }^{\circ} \mathrm{C}\right)\end{array}$ & $\begin{array}{l}\text { Reaction } \\
\text { time } \\
(\mathrm{h})\end{array}$ & $\begin{array}{l}\text { Melt } \\
\text { point } \\
\left({ }^{\circ} \mathrm{C}\right)\end{array}$ & $M_{\mathrm{n}}$ & $\begin{array}{l}\text { Polydispersity } \\
\text { index }\end{array}$ \\
\hline Samples & 420 & 4 & $<20$ & 552 & 1.6 \\
PCS-2 & 450 & 2 & $104-124$ & 1008 & 2.7 \\
PCS-6 & 450 & 6 & $158-175$ & 1505 & 2.6 \\
PCS-8 & 450 & 8 & $180-200$ & 1679 & 2.9 \\
PCS-10 & 450 & 10 & $216-241$ & 1758 & 3.4 \\
PCS-16 & 450 & 16 & $256-287$ & 2275 & 4.1 \\
PCS-24 & 450 & 24 & $>300$ & 2661 & 5.3
\end{tabular}

In the PDMS pyrolysis rearrangement conversion processes, the first reaction involved the pyrolysis of the $\mathrm{Si}-\mathrm{Si}$ bond in the PDMS and the rearrangement of the Si-Si bond to form a Si-C bond. When the Si-C bond becomes the main chain structure, the reactant converts to low-molecular-weight PCS. Then, PCS with a certain degree of polymerisation is attained by the rapid increase in the $M_{\mathrm{n}}$ by dehydrogenation and demethane condensation at $420-460^{\circ} \mathrm{C}$. PCS with good spinnability, which can be used as a precursor for the preparation of continuous $\mathrm{SiC}$ fibres, is the product of a particular condition in this process. The whole reaction process could be shown by the products obtained at $450{ }^{\circ} \mathrm{C}$ but with different holding times. Under different conditions, the molecular weight distribution of the reaction product changes as shown in Fig. 2 . The properties of the products synthesised under different conditions are listed in Table 1. The PCS- $x$ in the picture refers to the preparation condition of the sample is $x \mathrm{~h}$ at $450{ }^{\circ} \mathrm{C}$.

The LPS forms a low-molecular-weight polycarbosilane LPCS with a "unimodal" distribution with $M_{\mathrm{n}}$ of 552 and softening point lower than room temperature through a structural rearrangement reaction (Fig. 2b). A new peak appears on the lefthand side in a further reaction, becoming a bimodaldistribution PCS with $M_{\mathrm{n}}$ of 1008 , softening point of 104$124{ }^{\circ} \mathrm{C}$, and polydispersity index of 1.6 (Fig. 2c). With the prolongation of the reaction time from $4 \mathrm{~h}$ to $10 \mathrm{~h}$, the $M_{\mathrm{n}}$ increases from 1008 to 1758, the soft point increases from 104$124{ }^{\circ} \mathrm{C}$ to $216-241{ }^{\circ} \mathrm{C}$, and the polydispersity index increases from 1.6 to 3.4, with an increase in the high-molecular-weight peaks (Fig. 2c-f). As the reaction goes on further, the $M_{\mathrm{n}}$ increases from 1758 to 2661, the soft point increases from 216$241{ }^{\circ} \mathrm{C}$ to a temperature beyond the measurement range (300 ${ }^{\circ} \mathrm{C}$ ) and the polydispersity index increases from 3.4 to 5.2, with significant bulging on the left, but overall, they continued to exhibit a "bimodal" distribution (Fig. 2f-h). The GPC curve of the above PCS was also subjected to the same peak-splitting processing. The result is shown in Fig. 3.

Although the PCSs with different reaction degrees produce different GPC curves, the fitting curves obtained from the peaksplitting are in good agreement with the original curves, indicating that such peak processing is reasonable. It can also be seen that the original "unimodal" LPCS contains two peaks
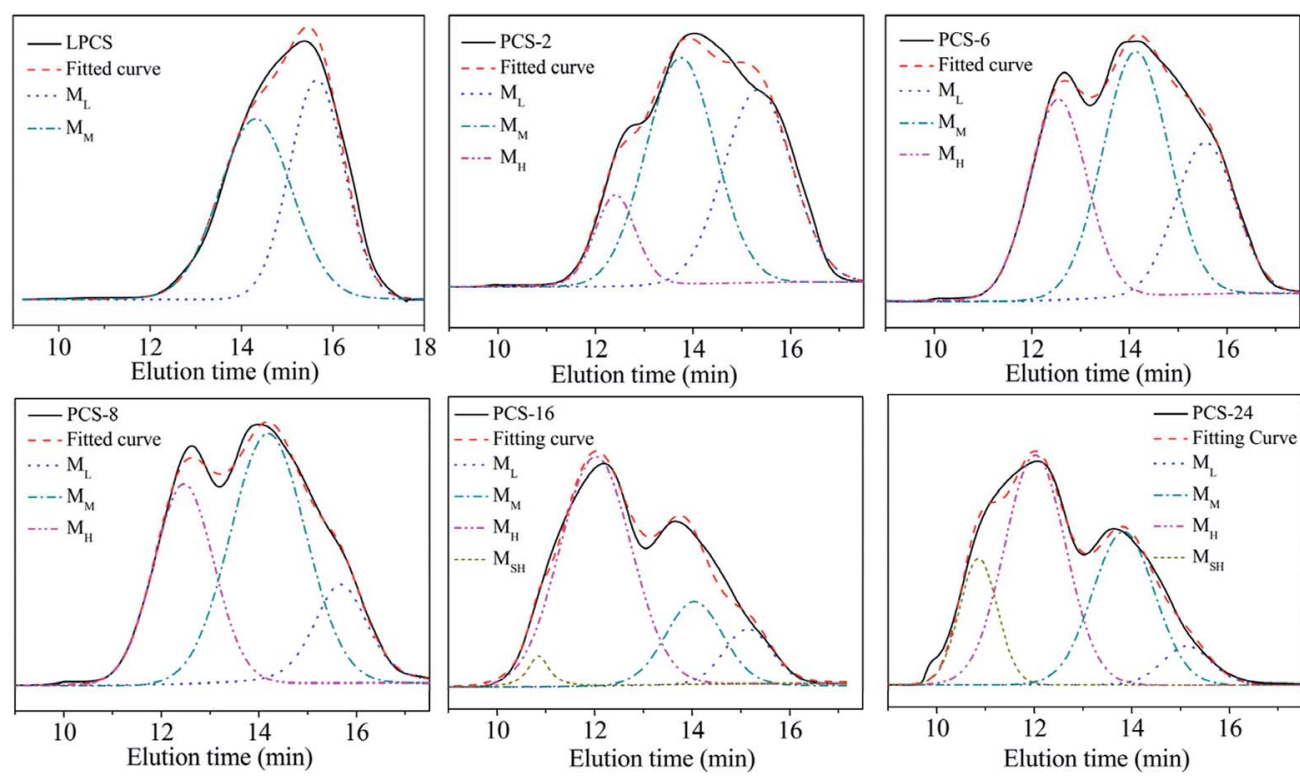

Fig. 3 GPC curves and fitting results of PCS synthesised under different conditions. 
Table 2 Peak positions in GPC curves of PCS synthesised under different conditions

\begin{tabular}{lllll}
\hline & \multicolumn{3}{l}{ Elution time (min) } & \\
\cline { 2 - 5 } Samples & $\mathrm{M}_{\mathrm{L}}$ & $\mathrm{M}_{\mathrm{M}}$ & $\mathrm{M}_{\mathrm{H}}$ & $\mathrm{M}_{\mathrm{SH}}$ \\
\hline LPCS & 15.7 & 14.2 & - & - \\
PCS-2 & 15.3 & 13.7 & 12.4 & - \\
PCS-6 & 15.6 & 14.1 & 12.5 & - \\
PCS-8 & 15.7 & 14.2 & 12.5 & - \\
PCS-10 & 15.3 & 14.0 & 12.4 & - \\
PCS-16 & 15.1 & 13.8 & 12.0 & 10.9 \\
PCS-24 & 15.1 & 13.8 & 12.0 & 10.9 \\
& & & & \\
\hline
\end{tabular}

while the original "bimodal" distribution of PCS-2, PCS-6, PCS8, and PCS-10 contains three peaks. Meanwhile, PCS-16 and PCS-24 exhibit a "bimodal + bulge" distribution, which means another new peak with an ultrahigh molecular weight $\left(\mathrm{M}_{\mathrm{SH}}\right)$ at $t$ $=10.9 \mathrm{~min}$ on the high-molecular-weight side is produced, that is, it actually has four peaks. The peak positions corresponding to each peak formed after these PCSs were fractionated are listed in Table 2.

As shown in the table, a low-molecular-weight peak $\mathrm{M}_{\mathrm{L}}$ and middle-molecular-weight peak $\mathrm{M}_{\mathrm{M}}$ appeared at $15.4 \mathrm{~min}$ and $14.0 \mathrm{~min}$ in each group of samples. With the increase in the reaction time, the higher-reacting PCS component produced a high-molecular-weight peak at $12.3 \operatorname{min~}_{\mathrm{H}}$, and an ultra-high molecular PCS component in the vicinity of $10.9 \mathrm{~min}$. Considering that all peaks occur at $15.4 \pm 0.3,14.0 \pm 0.3,12.3 \pm 0.3,9.7$, the peaks appearing at same position can be regarded as being of the same molecular type. This indicates that, during the hightemperature polycondensation to form PCS, specific molecular weight groups of different molecules will be formed. Specifically, the LPCS transformed by LPS contains both $\mathrm{M}_{\mathrm{L}}$ and $\mathrm{M}_{\mathrm{M}}$ molecules, while PCS-2, PCS-6, PCS-8, and PCS-10 contain the same $\mathrm{M}_{\mathrm{L}}$, $\mathrm{M}_{\mathrm{M}}$, and emerging $\mathrm{M}_{\mathrm{H}}$ molecules. The higher-molecular-weight PCS-16 and PCS-24 contain $\mathrm{M}_{\mathrm{L}}, \mathrm{M}_{\mathrm{M}}, \mathrm{M}_{\mathrm{H}}$, and new $\mathrm{M}_{\mathrm{SH}}$ molecules.

\subsection{Evolution of PCS components}

As shown in Fig. 2, in the synthesis of PCS, as the reaction proceeds, the molecular weight distribution of the product changes significantly. On the surface, it changes from

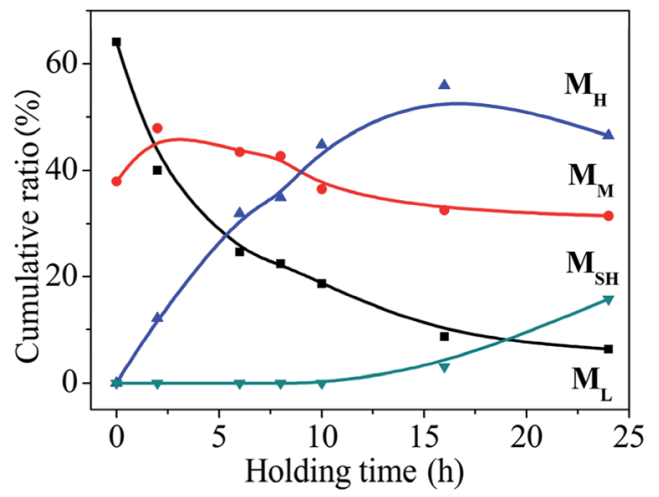

Fig. 4 Change in cumulative ratio of PCS with reaction time. "unimodal" to "double peak". After the peak-splitting treatment, the $\mathrm{M}_{\mathrm{L}}$ and $\mathrm{M}_{\mathrm{M}}$ molecules are actually produced first, followed by a polycondensation reaction between the two components to derive the third component which further reacts to generate the fourth component. The ratio of each component in the reaction at $450{ }^{\circ} \mathrm{C}$ at different times was quantitatively analysed, and the change in the mass fraction is shown in Fig. 4 (the initial sample was LPCS).

As the reaction time is prolonged, among the low-, medium-, and high-molecular-weight components, $\mathrm{M}_{\mathrm{L}}$ decreases rapidly and $\mathrm{M}_{\mathrm{M}}$ decreases slightly and then decreases slowly. Corresponding to the rapid decrease in the $\mathrm{M}_{\mathrm{L}}$ content, the $\mathrm{M}_{\mathrm{H}}$ content increases rapidly, reaching its highest value at about $16 \mathrm{~h}$. The reaction from the beginning of the reaction to about $16 \mathrm{~h}$ constitutes the first stage of the reaction. The LPCS formed by the rearrangement of the LPS contains only two components, that is, $\mathrm{M}_{\mathrm{L}}$ and $\mathrm{M}_{\mathrm{M}}$, with contents of $64.1 \%$ and $37.9 \%$, respectively. At this time, the $\mathrm{M}_{\mathrm{L}}$ is the main component. However, when the LPCS reacted at $450{ }^{\circ} \mathrm{C}$, the $\mathrm{M}_{\mathrm{L}}$ content began to decrease. After reacting for $2 \mathrm{~h}$, a new peak appeared at around $12.4 \mathrm{~min}$, indicating that the reaction had produced a new component, $\mathrm{M}_{\mathrm{H}}$. With the prolongation of the reaction time, the $\mathrm{M}_{\mathrm{L}}$ content decreased while $\mathrm{M}_{\mathrm{H}}$ increased significantly and $\mathrm{M}_{\mathrm{M}}$ decreased slowly. In the reaction, over about 2-10 h, the three components exhibited a "three pillars" trend. Once the reaction had run for about $16 \mathrm{~h}$, the $\mathrm{M}_{\mathrm{H}}$ no longer increased, marking the completion of the first stage of the reaction. In the first stage, the $\mathrm{M}_{\mathrm{L}}$ and $\mathrm{M}_{\mathrm{M}}$ are the main reactants, in which $\mathbf{M}_{\mathrm{M}}$ has both formation and consumption processes. After $16 \mathrm{~h}$, the reaction enters the second stage. The main characteristic of this phase is that the $\mathrm{M}_{\mathrm{L}}, \mathrm{M}_{\mathrm{M}}$, and $\mathrm{M}_{\mathrm{H}}$ contents all decrease as the reaction time increases, and another new component, $\mathrm{M}_{\mathrm{SH}}$, appears on the GPC chart. In this case, the resulting PCS is characterised by the original three components being replaced by four components, namely, $\mathrm{M}_{\mathrm{L}}, \mathrm{M}_{\mathrm{M}}, \mathrm{M}_{\mathrm{H}}$, and $\mathrm{M}_{\mathrm{SH}}$.

During the transition from the high-temperature polycondensation of LPCS to PCS, the evolution of the 2-3-4 components in the two stages of $M_{\mathrm{n}}$ increase is related to the reaction mechanism of the high-temperature polycondensation reaction. After the $\mathrm{Si}-\mathrm{Si}$ rearrangement reaction is completed at $400-420{ }^{\circ} \mathrm{C}$, the dehydrogenation and demethanisation reactions occur mainly at higher temperatures $\left(440-460{ }^{\circ} \mathrm{C}\right)$ :

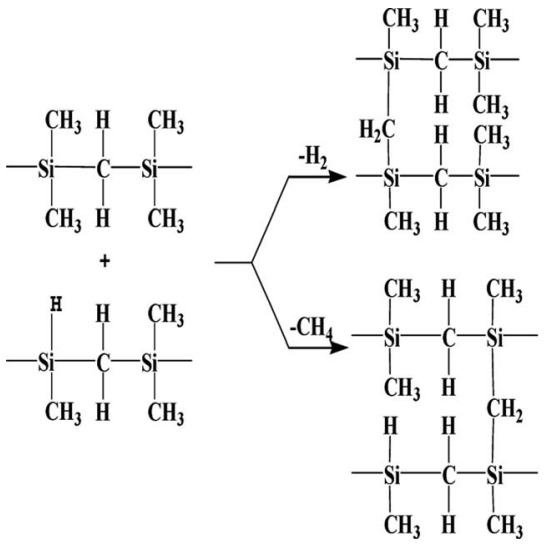


Table 3 Peak positions and $M_{n}$ for PCS obtained under different distillation conditions

\begin{tabular}{|c|c|c|c|c|c|c|}
\hline \multirow[b]{2}{*}{ Samples } & \multicolumn{2}{|c|}{ Distillation condition } & \multicolumn{2}{|l|}{ Distillation results } & \multicolumn{2}{|l|}{ Peak fitting results } \\
\hline & Temperature $\left({ }^{\circ} \mathrm{C}\right)$ & Time (h) & Peak position (min) & $M_{\mathrm{n}}$ & Peak position (min) & $M_{\mathrm{n}}$ \\
\hline${ }^{\prime} \mathrm{M}_{\mathrm{L}}$ & 360 & 2 & 15.7 & 393 & 15.6 & 531 \\
\hline${ }^{\prime} \mathbf{M}_{\mathrm{M}}$ & 420 & 2 & 14.3 & 1009 & 14.1 & 1207 \\
\hline${ }^{\prime} \mathrm{M}_{\mathrm{H}}$ & 440 & 5 & 12.8 & 2378 & 12.5 & 2731 \\
\hline
\end{tabular}
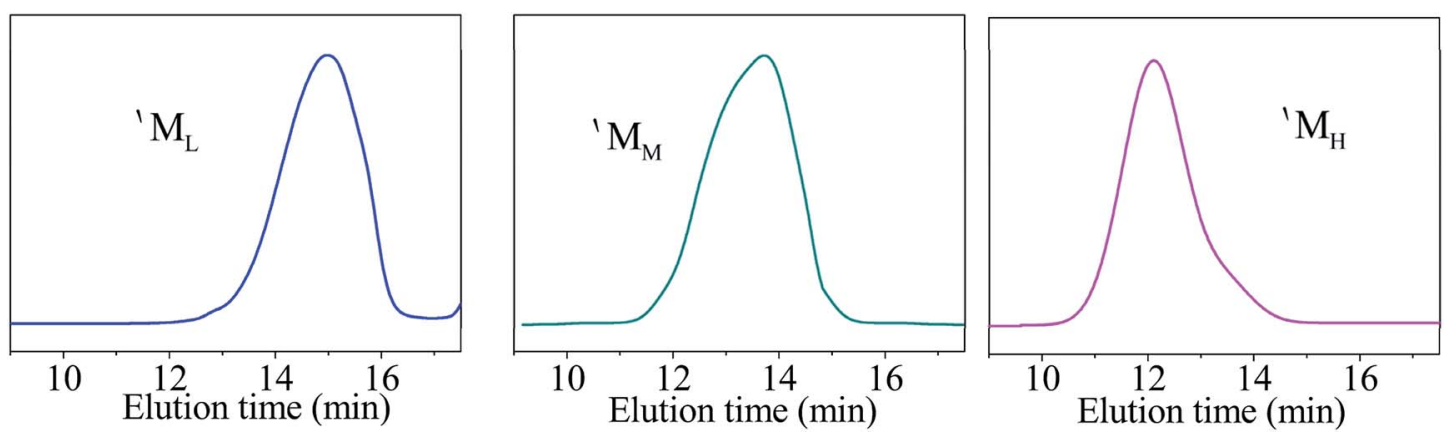

Fig. 5 GPC curves of distillation products.

This polycondensation reaction between molecules will inevitably bring about a rapid increase in the $M_{\mathrm{n}}$, and eventually form a complex structure with PCS of a certain $M_{\mathrm{n}}$. The hightemperature polycondensation process involves four different molecular weight components, namely, $\mathrm{M}_{\mathrm{L}}, \mathrm{M}_{\mathrm{M}}, \mathrm{M}_{\mathrm{H}}$, and $\mathrm{M}_{\mathrm{SH}}$, with the corresponding peak $M_{\mathrm{n}}$ being $531,1207,2731$, and 10977 . This variation points to the reactivity of the components varying from strong to weak in the order of $M_{L}>M_{M}>M_{H}>$ $\mathrm{M}_{\mathrm{SH}}$. As the peak value for a component falls, so too does its reaction activity. It thus takes more time or a greater amount of content to promote the reaction. Given the $M_{\mathrm{n}}$ values, it can be speculated that an intermolecular polycondensation reaction occurred between the main components, as follows:

First stage:

$$
\begin{aligned}
& 2 \mathrm{M}_{\mathrm{L}} \rightarrow \mathrm{M}_{\mathrm{M}} \\
& \mathrm{M}_{\mathrm{L}}+\mathrm{M}_{\mathrm{M}} \rightarrow \mathrm{M}_{\mathrm{H}} \\
& 2 \mathrm{M}_{\mathrm{M}} \rightarrow \mathrm{M}_{\mathrm{H}}
\end{aligned}
$$

Second stage:

$$
\mathrm{M}_{\mathrm{L}}+\mathrm{M}_{\mathrm{M}}+\mathrm{M}_{\mathrm{H}}+\cdots \rightarrow \mathrm{M}_{\mathrm{SH}}
$$

In the first stage, the polycondensation mainly occurs between two low-molecular-weight components, one lowmolecular-weight component and one middle-molecularweight component, or two middle-molecular-weight components. In the second stage, as more molecules become involved in the response, the polycondensation reaction occurs between multiple molecules. As mentioned above, since the reaction first forms two types of molecules, $\mathrm{M}_{\mathrm{L}}$ and $\mathrm{M}_{\mathrm{M}}$, having a specific $M_{\mathrm{n}}$, and then, in a subsequent reaction, forms $\mathrm{M}_{\mathrm{H}}$ and $\mathrm{M}_{\mathrm{SH}}$ molecules of a specific $M_{\mathrm{n}}$ according to a certain rule, there are no disorderly or diverse molecule species. Based on this evolution of $M_{\mathrm{n}}$ growth, the reaction conditions can be adjusted to control the composition of the product, resulting in a product with an appropriate structure and $M_{\mathrm{n}}$.

\subsection{Relationship between components and spinnability}

The above study shows that PCS with good spinnability is composed of $\mathrm{M}_{\mathrm{L}}, \mathrm{M}_{\mathrm{M}}, \mathrm{M}_{\mathrm{H}}$. Therefore, these three components and their impact on spinnability need to be studied. In addition, the above-mentioned peak-splitting processing is very good for dividing good-spinnability PCS into three components with a normal distribution, but this is done through image processing, and whether these three components actually exist in PCS remains questionable. There is, therefore, a need for experimental separation to prove this. To isolate the PCS components, several researchers previously used solvent extraction or chromatographic column fractionation., ${ }^{8,9}$ However, it is difficult to accurately separate the components by means of solvent extraction, which is accompanied by some molecular weight tailing in the separated components. The column separation method is inefficient and can only produce small amounts of samples, which cannot be used to test their spinnability. In the present study, a distillation unit, designed and built in-house, was used to perform the vacuum distillation of PCS-6 under different conditions. By controlling the distillation temperature, the three components with low, medium, and high $M_{\mathrm{n}}$ (denoted ${ }^{\prime} \mathrm{M}_{\mathrm{L}},{ }^{\prime} \mathrm{M}_{\mathrm{M}}$, and ${ }^{\prime} \mathrm{M}_{\mathrm{H}}$ ) were separated from the PCS- 6 . The vacuum distillation conditions and the properties of the three obtained fractions are listed in Table 3, in which ${ }^{\prime} \mathrm{M}_{\mathrm{L}}$ and ${ }^{\prime} \mathrm{M}_{\mathrm{M}}$ correspond to the fractions obtained from the distillation, and ${ }^{\prime} \mathrm{M}_{\mathrm{H}}$ to the fraction remaining after the 
Table 4 Spinnability of $M_{L}, M_{M}$, and $M_{H}$

\begin{tabular}{|c|c|c|c|c|c|}
\hline Samples & $\begin{array}{l}\text { Melt point } \\
\left({ }^{\circ} \mathrm{C}\right)\end{array}$ & $\begin{array}{l}\text { De-foaming temperature } \\
\left({ }^{\circ} \mathrm{C}\right)\end{array}$ & $\begin{array}{l}\text { Optimum spinning } \\
\text { temperature }\left({ }^{\circ} \mathrm{C}\right)\end{array}$ & $\begin{array}{l}\text { Average continuous spinning } \\
\text { time (min) }\end{array}$ & $\begin{array}{l}\text { Diameter } \\
(\mu \mathrm{m})\end{array}$ \\
\hline $\mathrm{M}_{\mathrm{L}}$ & $<30$ & 150 & - & 0 & - \\
\hline $\mathrm{M}_{\mathrm{H}}$ & $>300$ & 410 & - & $<2$ & - \\
\hline
\end{tabular}

Table 5 Melt point, composition ratio, and spinnability of PCS

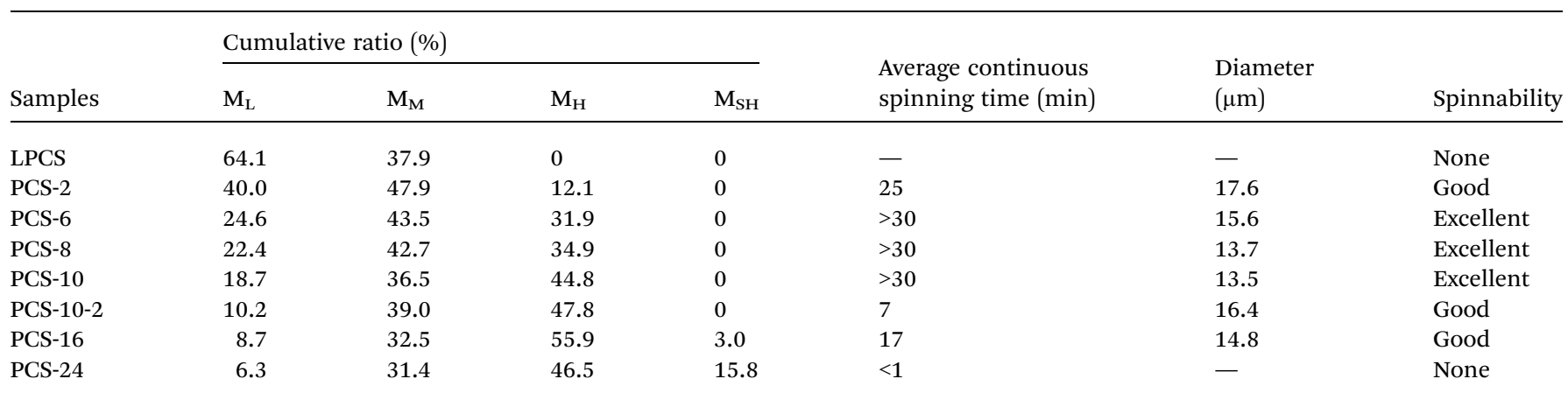

distillation. For comparison, the corresponding peak-splitting results for $\mathrm{M}_{\mathrm{L}}, \mathrm{M}_{\mathrm{M}}$, and $\mathrm{M}_{\mathrm{H}}$, obtained from the PCS-6, are also listed in the table. The GPC for ${ }^{\prime} \mathrm{M}_{\mathrm{L}},{ }^{\prime} \mathrm{M}_{\mathrm{M}}$, and ${ }^{\prime} \mathrm{M}_{\mathrm{H}}$ is shown in Fig. 5.

Although the peak shape of the three components separated from PCS-6 by vacuum distillation is not completely normal, and there is still some tailing in $\mathrm{M}_{\mathrm{H}}$, the overall composition is a single peak, which is better than the result attained when using solvent extraction. The peak positions of the three fractions were $12.8,14.3$ and $15.7 \mathrm{~min}$, respectively, corresponding to and closely approximating to the $12.5,14.1$, and $15.6 \mathrm{~min}$ of the PCS-6 peak-splitting value, and the corresponding $M_{\mathrm{n}}$ deviation was not large. The three samples are equivalent to the $\mathrm{M}_{\mathrm{L}}, \mathrm{M}_{\mathrm{M}}$, and $\mathrm{M}_{\mathrm{H}}$ components ( $\mathrm{M}_{\mathrm{L}},{ }^{\prime} \mathrm{M}_{\mathrm{M}}$, and ${ }^{\prime} \mathrm{M}_{\mathrm{H}}$ are expressed as $\mathrm{M}_{\mathrm{L}}, \mathrm{M}_{\mathrm{M}}$, and $\mathrm{M}_{\mathrm{H}}$ in the following discussion). This also shows that the above-mentioned results of the peak-splitting are correct, demonstrating that the above three components are present in the PCS.

As mentioned above, during the synthesis of the PCS, the intermolecular polycondensation reaction takes place during the conversion of the components from $\mathrm{M}_{\mathrm{L}}$ to $\mathrm{M}_{\mathrm{M}}$ and from $\mathrm{M}_{\mathrm{M}}$ to $\mathrm{M}_{\mathrm{H}}$. In addition to causing a doubling of the $M_{\mathrm{n}}$ of the components, this also causes changes in the molecular structure (ESI, Fig. S1-S4, Tables S1 and S2 $\dagger$ ). In particular, the occurrence of intramolecular cyclisation and a branched structure will significantly change the molecular structure and thus affect the spinnability of the product. As the linearity of the molecule increases, the rheology improves, as does the spinnability. The linearity increases with the increase in the length of the molecular chain and decreases with any increase in the ring and branching structures. In the transformation of the components, the existing molecular chain, and the ring and branching structures increase, which will have some impact on the spinnability of the PCS. To determine the spinnability of the three components, they were individually placed in a singlehole melt spinning apparatus, which was used to conduct spinning at a constant spinning pressure of $0.3 \mathrm{MPa}$ and a takeup rate of $267 \mathrm{~m} \mathrm{~min}^{-1}$. The results are listed in Table 4 .

The $\mathrm{M}_{\mathrm{L}}$ has a low $M_{\mathrm{n}}$, and a softening point that is lower than room temperature, such that it is a viscous liquid at room temperature. When spun at room temperature, the sample drips after being extruded from the spinneret and cannot be formed into fibres. The spinnability of the $\mathrm{M}_{\mathrm{M}}$ component is better than that of $\mathrm{M}_{\mathrm{L}}$. Continuous spinning can be achieved at an appropriate de-foaming temperature and spinning temperature, but the spinnability is still inferior to that of commercial PCS (PCS-10). $\mathrm{M}_{\mathrm{H}}$ has a higher $M_{\mathrm{n}}$ than $\mathrm{M}_{\mathrm{M}}$, so may have a better spinnability. However, due to its high softening point, defoaming in the melting cylinder at $410{ }^{\circ} \mathrm{C}$ for $2 \mathrm{~h}$ will still fail to completely de-foam the sample, such that when the melt is spun from the spinneret, the extrusion will be intermittent, failing to produce a continuous fibre. Increasing the de-foaming temperature will cause further polycondensation, resulting in increased viscosity, and thus the failure of the spinning.

The above results indicate that the spinnability of PCS is not determined by a single component, but that it is a combined effect of all the components. An analysis of the evolution process in the last section of the PCS reveals that two components, three components, and four components, as well as three kinds of PCS, were formed during the reaction. To verify the contribution and influence of each component on the spinning, spinning experiments were undertaken with these three types of PCS, as shown in Table 5 (spinning conditions and pictures of fibers were shown respectively in the Table S3 and Fig. S5 in ESI†). PCS-10-2 sample obtained after 30 min re-vacuum of PCS10.

The effect of the four components on the spinnability of the PCS is shown in the table: LPCS containing only the $\mathrm{M}_{\mathrm{L}}$ and $\mathrm{M}_{\mathrm{M}}$ 
components incurs the same problem as $\mathrm{M}_{\mathrm{L}}$ in the spinning process because $\mathrm{M}_{\mathrm{L}}$ is the main component. The sample drips after being extruded from the spinneret and cannot be formed into fibres. PCS- 2 to PCS-10, all containing $\mathrm{M}_{\mathrm{L}}, \mathrm{M}_{\mathrm{M}}$, and $\mathrm{M}_{\mathrm{H}}$, exhibited good or excellent spinnability, and as the value of $\mathbf{M}_{H}$ increased, the diameter of the spun fibre decreased from 17.6 to $13.5 \mu \mathrm{m}$. As the $\mathbf{M}_{\mathrm{H}}$ content of the PCS increased, its spinnability further improved, indicating that, among the three PCS components, the $\mathrm{M}_{\mathrm{H}}$ structure is the most suitable for spinning, and makes the largest contribution to the spinnability. When the higher $M_{\mathrm{n}} \mathrm{M}_{\mathrm{SH}}$ components in the PCS formed a fourcomponent system, the spinnability gradually decreased in order, from PCS-16 to PCS-24.

Obviously, the spinnability of the PCS depends on its composition. The $M_{\mathrm{n}}$, structure and content of each component have different impacts on the spinnability of the PCS. The $M_{\mathrm{n}}$ and structure of $\mathrm{M}_{\mathrm{H}}$ are suitable and are key to determining the spinnability of PCS. Although $\mathrm{M}_{\mathrm{H}}$ is not suitable for melt-spinning by itself, it exhibits excellent spinnability when combined with $\mathrm{M}_{\mathrm{L}}$ and $\mathbf{M}_{\mathbf{M}}$. When $\mathbf{M}_{\mathrm{SH}}$ is added, however, since $\mathbf{M}_{\mathrm{SH}}$ is a polymolecular polycondensation reaction product with a $M_{\mathrm{n}}$ that is four times greater than that of $\mathrm{M}_{\mathrm{H}}$, the structure contains more cyclised, branched, and even crosslinked structures, thus adversely affecting the spinnability of PCS. Of the three components of PCS, $\mathrm{M}_{\mathrm{L}}$ exhibits no spinnability but is nevertheless an indispensable component. The $\mathrm{M}_{\mathrm{L}}$ content in PCS-10 (which has good spinnability) was reduced by about $8 \%$, thus adversely affecting the spinnability of the PCS. Although the drastic reduction in the $\mathrm{M}_{\mathrm{L}}$ content from PCS-2 to PCS-10 does not significantly affect the spinnability, there is clearly a limit to the effect on the spinnability when the $\mathrm{M}_{\mathrm{L}}$ content is below this limit. The presence of $\mathrm{M}_{\mathrm{L}}$ lowers the softening point of the PCS and can also act as a lubricant at the spinning temperature, which avoids the selfpolymerisation of the samples, ensures good de-foaming, and reduces the dependence of the PCS viscosity on the temperature, making the viscosity temperature curve smoother and the spinning process more stable.

The above studies show that a "unimodal" type of LPCS containing both $\mathrm{M}_{\mathrm{L}}$ and $\mathrm{M}_{\mathrm{M}}$ components is not suitable as a raw material for spinning due to its $M_{\mathrm{n}}$ and low softening point; the "bimodal + bulge" four-component PCS exhibits poor spinnability due to the presence of the $\mathrm{M}_{\mathrm{SH}}$ component. Only the three-component PCS offers excellent spinnability. In the threecomponent $\left(\mathrm{M}_{\mathrm{L}}, \mathrm{M}_{\mathrm{M}}\right.$, and $\left.\mathrm{M}_{\mathrm{H}}\right)$ PCS, the $\mathrm{M}_{\mathrm{H}}$ provides a linear structure that realises the spinnability and which is a major contributor to the spinnability, while $\mathrm{M}_{\mathrm{L}}$ ensures uniform melting, de-foaming, and stable spinning. In the synthesis of spinning-grade PCS, the $\mathrm{M}_{\mathrm{H}}$ content must be increased as much as possible and the required $\mathrm{M}_{\mathrm{L}}$ content should be maintained to ensure a reasonable ratio of the three components. The optimal ratio, determined by experiment, was $30-45 \% \mathrm{M}_{\mathrm{H}}, 30-$ $51 \% \mathrm{M}_{\mathrm{M}}$, and $18-25 \% \mathrm{M}_{\mathrm{L}}$.

\section{Conclusions}

In the present study, the molecular composition of PCS, the evolution of the synthesis process, and the influence of each component on the spinnability were systematically studied by combining distillation separation with GPC peak-split. The results showed that, during the synthesis of PCS, LPCS contains two components, $\mathrm{M}_{\mathrm{L}}$ and $\mathrm{M}_{\mathrm{M}}$. Because of further hightemperature pyrolysis, two new components, $\mathbf{M}_{\mathrm{H}}$ and $\mathbf{M}_{\mathrm{SH}}$, were produced. The product composition evolves from two components, through three components, to four components. PCS with good spinnability has average $\mathrm{M}_{\mathrm{L}}, \mathrm{M}_{\mathrm{M}}$, and $\mathrm{M}_{\mathrm{H}} M_{\mathrm{n}}$ of 531, 1207, and 2731. The spinnability of the PCS does not depend on a single component but on the combined effect of the individual components. The $\mathbf{M}_{\mathrm{H}}$ polymer component provides a linear structure and makes the greatest contribution to the spinnability. The $\mathrm{M}_{\mathrm{L}}$ ensures the uniform melting and defoaming, as well as the stable spinning of the PCS. To produce PCS with good spinnability, the content of the $\mathbf{M}_{\mathrm{H}}$ polymer component should be maximised while an appropriate amount of the $\mathrm{M}_{\mathrm{L}}$ should be incorporated. Results of experiments proved that the optimum content ratios were $30-45 \% \mathrm{M}_{\mathrm{H}}, 30-$ $51 \% \mathrm{M}_{\mathrm{M}}$, and $18-25 \% \mathrm{M}_{\mathrm{L}}$.

\section{Conflicts of interest}

There are no conflicts to declare.

\section{Abbreviations}

$\begin{array}{ll}\mathbf{M}_{\mathrm{L}} & \text { Low-molecular-weights component } \\ \mathbf{M}_{\mathrm{M}} & \text { Middle-molecular-weights component } \\ \mathbf{M}_{\mathrm{H}} & \text { High-molecular-weights component } \\ \mathrm{M}_{\mathrm{SH}} & \text { Super-high-average-molecular-weights component } \\ \text { CMC } & \text { Ceramic-matrix composite } \\ \text { GPC } & \text { Gel permeation chromatogram } \\ \text { PCS } & \text { Polycarbosilane } \\ \text { SiC } & \text { Silicon carbide } \\ \text { THF } & \text { Tetrahydrofuran } \\ \text { PDMS } & \text { Polydimethylsilane } \\ \text { LPS } & \text { Liquid polysilane }\end{array}$

\section{Acknowledgements}

This work was supported by the Aid Program for Science and Technology Innovative Research Team in Higher Education Institutions of Hunan Province and by the Aid Program for Innovative Group of National University of Defense Technology.

\section{References}

1 F. W. Zok, Ceramic-matrix composites enable revolutionary gains in turbine engine efficiency, Am. Ceram. Soc. Bull., 2016, 95, 22-28.

2 K. Rivera, T. Muth, J. Rhoat, et al., Novel temperature sensors for SiC-SiC CMC engine components, J. Mater. Res., 2017, 32, 1-7.

3 A. Idesaki, M. Narisawa, K. Okamura and M. Sugimoto, Fine SiC fiber synthesized from organosilicon polymers: 
relationship between spinning temperature and melt viscosity of precursor polymers, J. Mater. Sci., 2001, 36, 5565-5569.

4 T. Nozawa, T. Hinoki, A. Hasegawa, et al., Recent advances and issues in development of silicon carbide composites for fusion applications, J. Nucl. Mater., 2009, 17, 622-627.

5 A. R. Bunsell and A. Piant, A review of the development of three generations of small diameter silicon carbide fibres, J. Mater. Sci., 2006, 41, 823-839.

6 Q. Yuan, Y. Li and Y. Song, Microstructure and thermal stability of low-oxygen SiC fibers prepared by an economical chemical vapor curing method, Ceram. Int., 2017, 43, 9128-9132.

7 K. Okada, Process for Producing Silicon Carbide Fiber, US Pat. 6316051, 2001-11-13.
8 Z. Chu, H. Liu, C. Feng, et al., Modulation of spinnable polycarbosilane with a high melting point via fractional precipitation, J. Natl. Univ. Def. Technol., 2004, 2, 39-43.

9 X. Cheng, Z. Xie, Y. Song and J. Xiao, The molecular weight distribution and spinnability of polycarbosilane synthesized under high pressure, Mater. Sci. Eng., 2005, 21, 59-62.

10 Y. Li and Y. Song, Synthesis and Spinnability of the High Softening-point Polycarbosilane, Chem. J. Chin. Univ., 2014, 35, 2272-2280.

11 G. Wang and Y. Song, Enhancing the Yield of Polycarbosilane Synthesis via Recycling of Liquid Byproduct at Atmospheric Pressure, Ceram. Int., 2018, 44, 6474-6478. 